Single Crystal Deformation Experiments for Validation of Dislocation Dynamics Simulations

D. H. Lassila, M. M. LeBlanc, M. Rhee

November 13, 2003

Materials Research Society Conference Proceedings 
This document was prepared as an account of work sponsored by an agency of the United States Government. Neither the United States Government nor the University of California nor any of their employees, makes any warranty, express or implied, or assumes any legal liability or responsibility for the accuracy, completeness, or usefulness of any information, apparatus, product, or process disclosed, or represents that its use would not infringe privately owned rights. Reference herein to any specific commercial product, process, or service by trade name, trademark, manufacturer, or otherwise, does not necessarily constitute or imply its endorsement, recommendation, or favoring by the United States Government or the University of California. The views and opinions of authors expressed herein do not necessarily state or reflect those of the United States Government or the University of California, and shall not be used for advertising or product endorsement purposes. 


\title{
Single Crystal Deformation Experiments for Validation of Dislocation Dynamics Simulations
}

David H. Lassila, Mary M. LeBlanc, and Moono Rhee

Lawrence Livermore National Laboratory, P.O. Box 808 L-113, Livermore, CA 94551-0808.

\begin{abstract}
A new experimental apparatus has been developed for performing compression deformation experiments on high-purity Mo single crystals. These experiments provide data that can validate 3-D dislocation dynamics (DD) simulations. The experiments are performed under conditions that allow unconstrained deformation; thus, a relatively uniform state of axial stress is maintained during deformation. In the following sections, we describe the new experimental apparatus and our results from experiments performed at ambient temperature at a strain rate of $\mathrm{s}^{-1}$. Validation criteria based on the Mo experiments may include comparing the stress-strain response using 3-D strain information, the predicted slip-system yield, and work-hardening behavior.
\end{abstract}

\section{INTRODUCTION}

In the past 10 years, there have been numerous developments in the area of 3-D, discrete DD simulation capabilities [1-5]. These computer codes can now simulate the stress-strain response (yield and work-hardening) and accompanying dislocation structure evolution of both facecentered cubic (fcc) and body-centered cubic (bcc) single crystals. At this time, the computer codes can simulate the deformation response to strains up to $1 \%$. The codes are generally more efficient in terms of computational speed when simulating high strain-rate deformation (e.g., strain rates of $\mathrm{s}^{-1}$ or greater).

In the case of bcc metals, a large body of experimental data exists [6,7]. Many interesting properties of bcc metals - such as their high strain-rate and temperature dependence of the yield strength, and their wavy glide at high temperatures - have been studied extensively. However, relatively few studies have reported the type of detailed information that is needed to rigorously validate DD simulations at the small strains that are of interest (i.e., on the order of 0.1 to $1.0 \%$ ). To effectively validate these simulations, the axial stress-strain response and as much information as possible about any slip activity during the deformation must be recorded.

When a single crystal deforms, the various slip-system activities typically result in irregular shape changes. In deformation experiments, these irregular shape changes can lead to nonuniformities in stress states in the crystal and/or a nonuniform strain field. Because of this possibility, researchers must take special care to understand both the crystal's stress state and shape change during deformation if they wish to collect the information needed to validate 3-D DD-simulation results.

In this work, we describe an experimental technique that was developed to minimize the nonuniformities in stress state that can occur during the deformation of single crystals. This technique was specifically developed to enable the validation of 3-D, discrete DD simulations. When using this technique, a deformation experiment is performed in compression; this allows essentially unconstrained deformation of single crystals that are oriented for a "single slip" under 
a condition of uniaxial stress. Several diagnostic techniques evaluate the crystal's shape change during deformation.

\section{SIX DEGREES OF FREEDOM (6 DOF) EXPERIMENT}

Our experiments involve the compression deformation of high-purity Mo single crystals at a nominal strain rate of $1 \mathrm{~s}^{-1}$ and under an axial strain of $0.6 \%$. The test sample used in this study has a square cross section that measures $5.5 \mathrm{~mm}$ on each side; the sample also has a length of $15 \mathrm{~mm}$, as shown in Figure 1. The experimental design is shown schematically in Figure 2. The upper platen is a half-sphere, which is point-loaded; this allows the single crystal to develop a tilt relative to the lower-platen surface during deformation. The lower platen rests on a set of ball bearings, thereby allowing the test sample bottom to translate and twist relative to the top platen with a minimal amount of frictional constraint. Including the degree of freedom gained through axial displacement, this design gives the deformation response of the single crystal six degrees of freedom. The goal of this design is to accommodate both (1) the resulting translation due to the flux of dislocation moving through the crystal and (2) the twist and tilt that results from the development of a dislocation structure without appreciably affecting the uniaxial stress state.

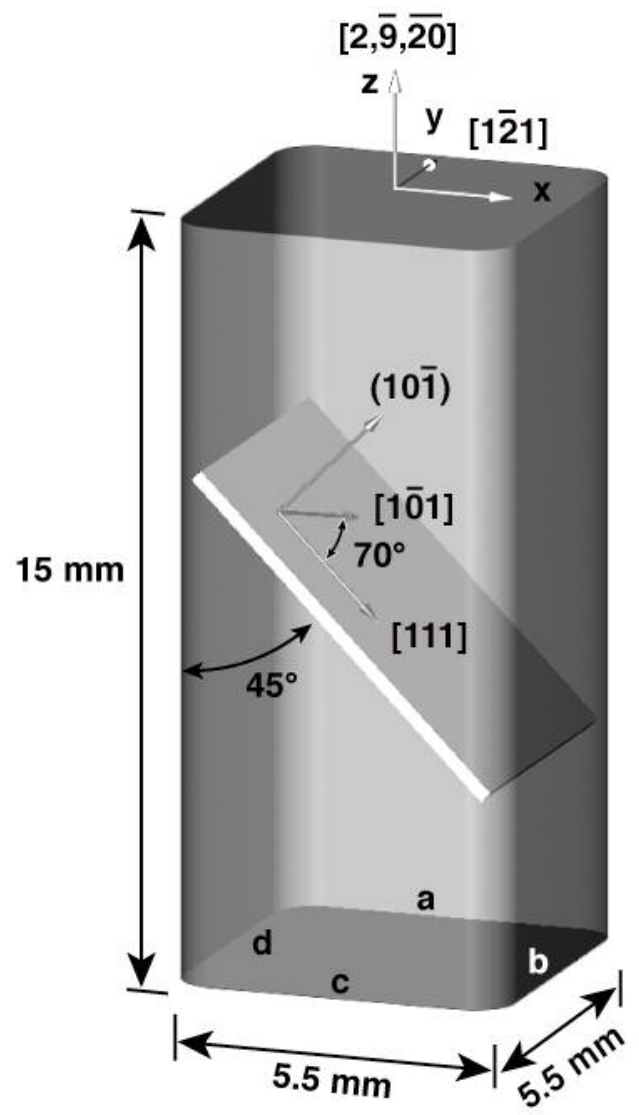

Figure 1. Single crystal test sample, as placed in the laboratory reference frame. The sample has a square cross section that measures $5.5 \mathrm{~mm}$ on each side and has a corner radius of $1 \mathrm{~mm}$. As shown, the $[2, \overline{9}, \overline{20}]$ direction points in the positive $z$ direction and the $[1 \overline{2} 1]$ direction points in the positive $y$ direction. The four faces of the sample are identified as "A" through "D." The primary slip plane and slip directions are also labeled. 


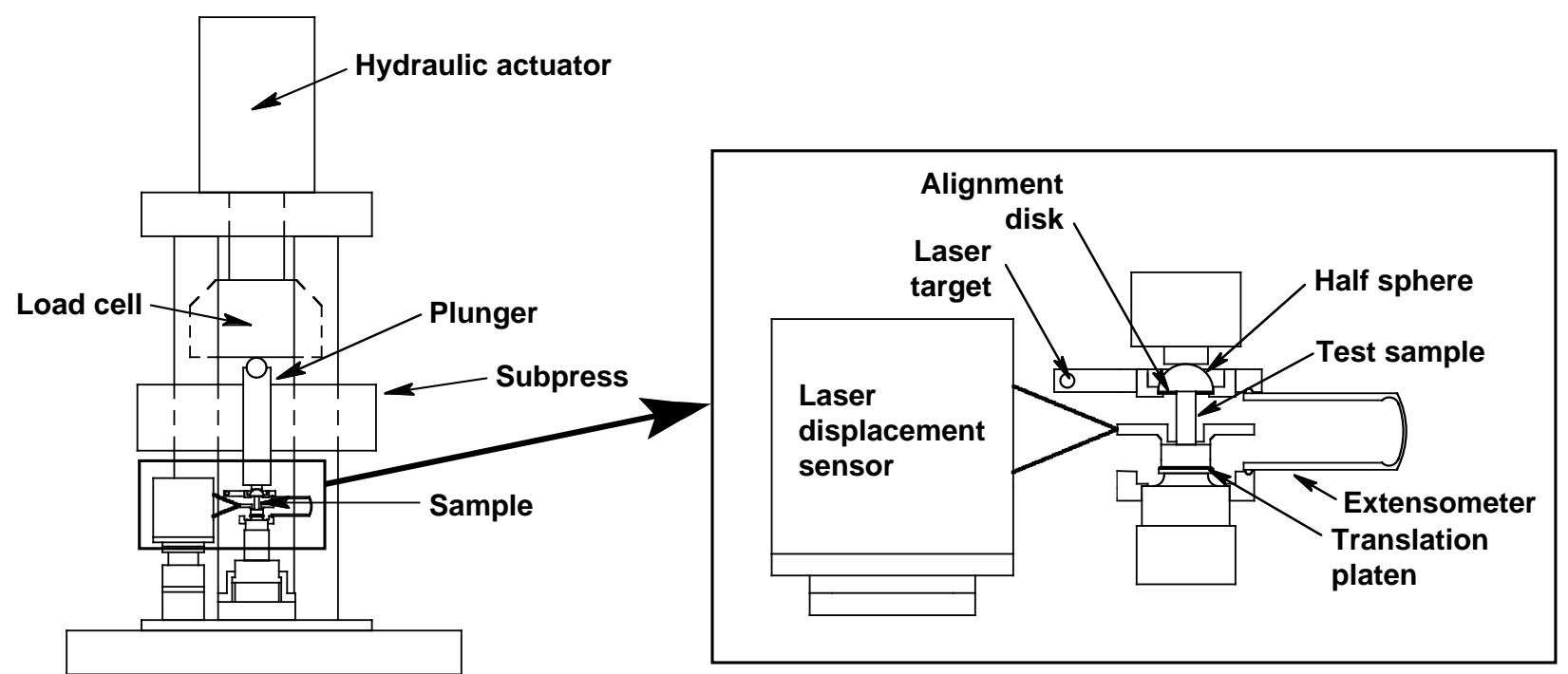

Figure 2. A schematic representation of the test apparatus. On the left is the overall view showing the loading actuator, load cell, and subpress. On the right is a detailed view of the sample, loading platen arrangement, and displacement sensors.

Three extensometers equally spaced around the half-sphere measure both the $z$-axis displacement and the tilt of the upper platen relative to the lower translation platen. Four laser displacement gages track the position of the lower translation platen. The measurement and position data are used to calculate the translation of the center of the lower platen relative to the center of the upper platen in the $x$ and $y$ coordinate directions, as well as the sample's twist about the $z$ axis.

We attached resistance-strain gage rosettes to the sides of one test sample. These rosettes measured the biaxial strain on the test sample surface during deformation. Each strain gage was attached with adhesive at the center of each side of the test sample. The gages were then wired in a Wheatstone bridge configuration.

\section{EXPERIMENTAL RESULTS}

Deformation experiments were conducted with displacement control such that a nominal axial strain rate of $1 \mathrm{~s}^{-1}$ was achieved. During deformation, 21 channels of test data were recorded using a variety of digital recorders. These data were subsequently downloaded into a computer for data analyses. The test results from two experiments involving different test samples (one attached with strain gages and another possessing polished surfaces for slip trace analyses) are reported here. We have conducted numerous experiments at the time of this writing and have found the test results to be very reproducible in terms of sample deformation (straingage response and platen motion) and the occurrence of slip traces.

The lower platen translation in the $x$ and $y$ directions during the axial deformation is shown in Figure 3. This plot indicates the relative motion of the center of the bottom of the test sample with respect to the center of the top in the laboratory reference frame. The ratio of the translations in the $x$ and $y$ directions is approximately constant, and the total displacement is $0.15 \mathrm{~mm}$. 


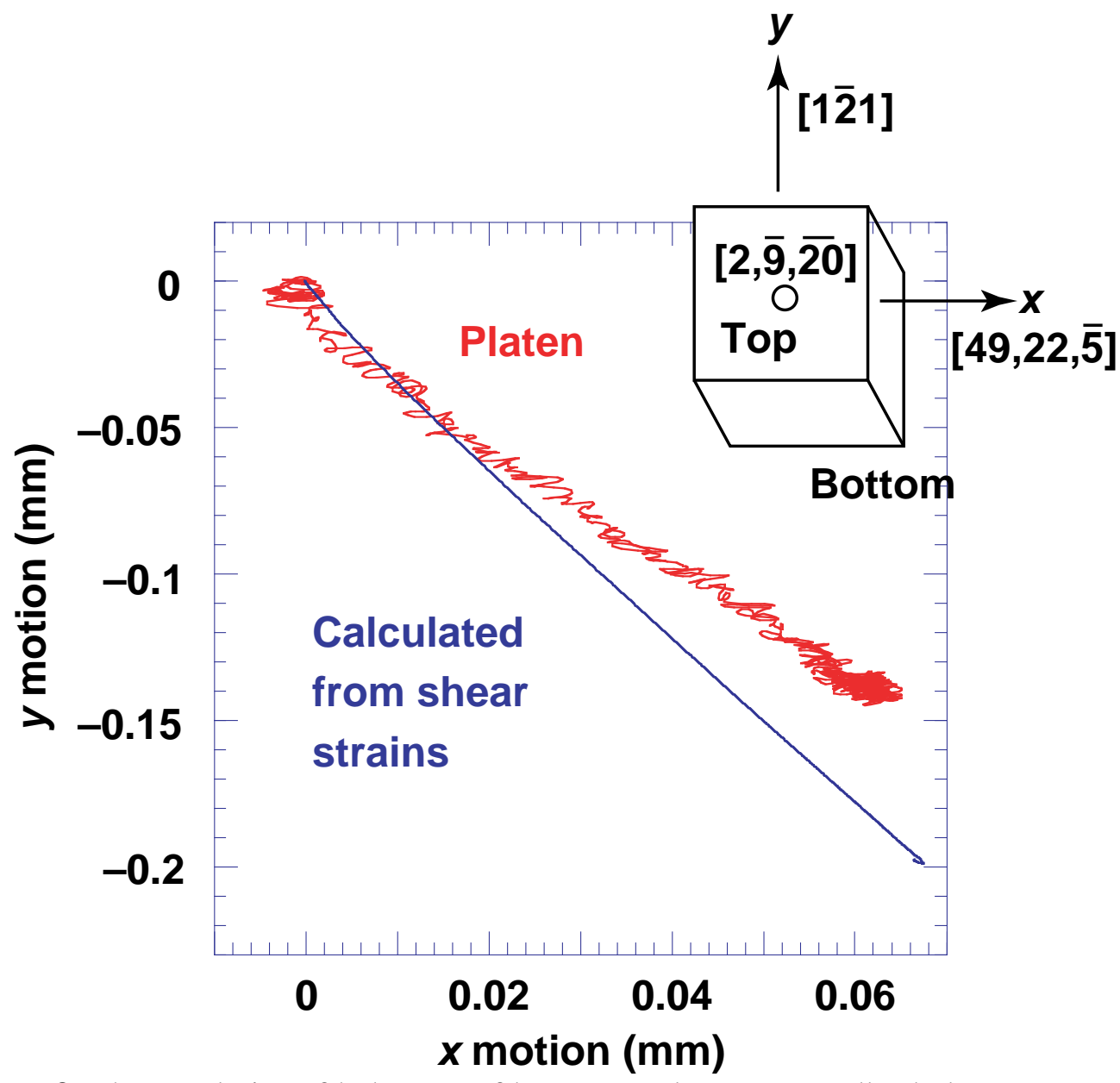

Figure 3. The translation of the bottom of the test sample as measured by the laser sensors. The total linear translation of the bottom of the sample was approximately $150 \mathrm{~mm}$ in a combination of the positive $x$ and negative $y$ directions.

We conducted conventional mechanics analyses with the raw data from the strain-gage rosettes to determine the biaxial state of elastic/plastic strain at the center locations on all four sides of the test sample. All strains were calculated with respect to the laboratory coordinate system. The axial $(z)$, lateral ( $x$ and $y$ ) and shear ( $x z$ and $y z)$ strains are plotted in Figure 4 . The $z$-axis strains are nearly equal on all four sides of the test sample, which is consistent with the small amount of measured tilt of the upper platen with respect to the lower platen. The three extensometers determined the tilt to be 0.1 degree at the maximum strain. 


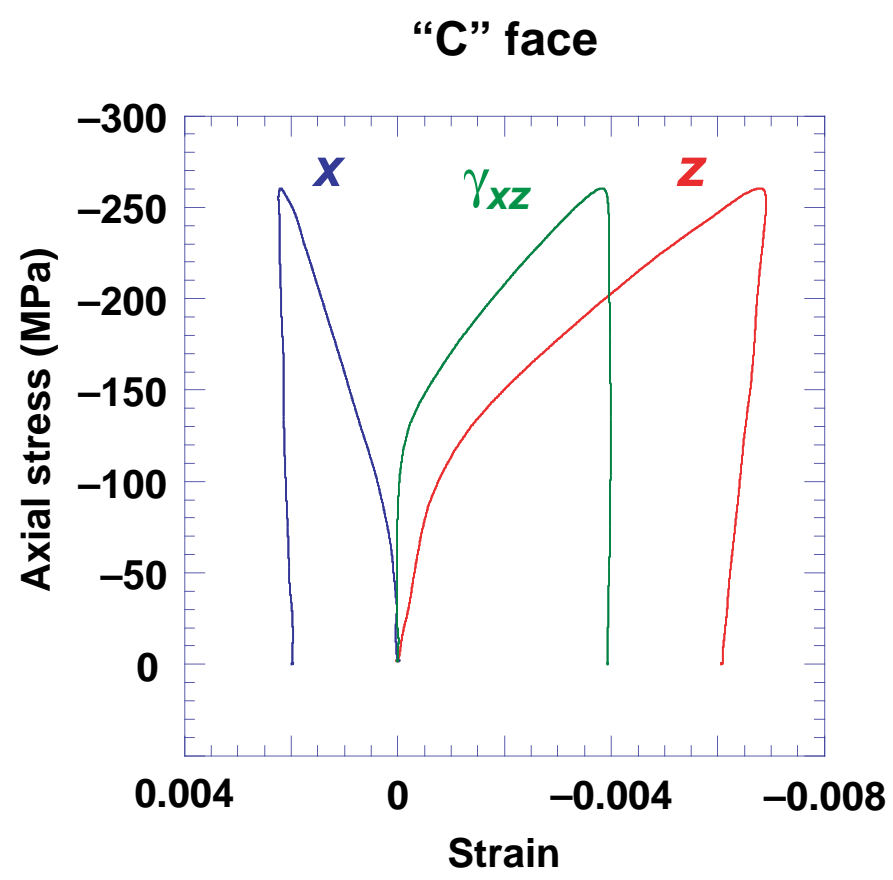

(a)
“B” face

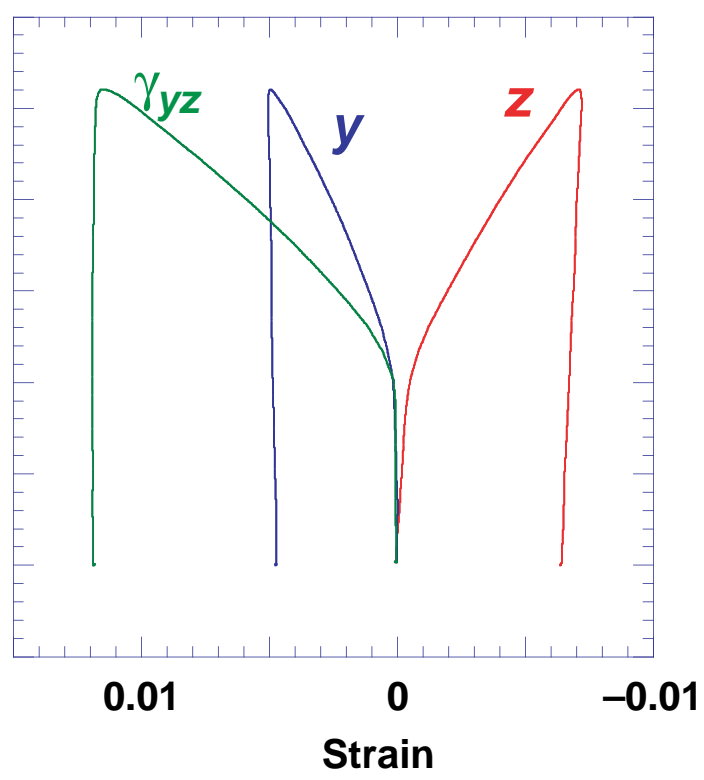

(b)

Figure 4. Stress-strain curves for a Mo crystal showing a comparison of the strains on opposite faces of the sample. As labeled, the strains shown are for the coordinate axis directions $x, y$, and $z$ and for the shear strains $\gamma_{x z}$ and $\gamma_{y z}$. The strains for the "C" and "A" faces are shown in (a) above. The strains for the "B" and "D" faces are shown in (b) above.

If a test sample deforms homogeneously in the absence of twist or tilt, the opposite faces will develop identical states of biaxial strain. As shown in Figure 4, the $z$-axis strains are within a few percent of one another, thereby showing little variation. The lateral strains $(x, y)$ and shear strains exhibit more variation on the order of 10 to $20 \%$. However, these variations appear to be consistent with the small measured amounts of twist and tilt. If we assume that the strain in the test sample is approximately uniform, an average value of the shear strains can be used to calculate the translation of the test sample bottom relative to the top in the $x$ and $y$ directions with the following formulas:

$$
\delta_{x}=\gamma_{z x} L \quad \text { and } \quad \delta_{y}=\gamma_{z y} L
$$

where $\gamma$ is the engineering shear strain and $L$ is the length of the test sample. The calculated translation of the lower platen based on the strain gage data is in good agreement with the translation measured using the laser displacement gages, as shown in Figure 3.

We tested a test sample identical to the one described above-except that the second test sample had polished sides (sans strain gages) — to conduct slip-trace analyses. We used optical light microscopy in conjunction with the Normarski interference contrast technique to observe

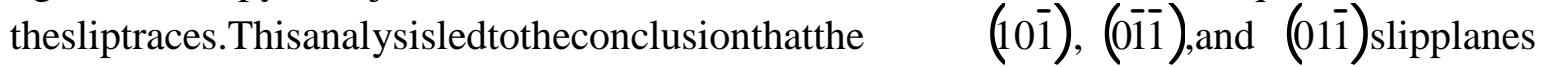
were active, as illustrated in Figure 5. Transmission electron microscopy (TEM) foils were 
prepared from one deformed sample. The TEM observations of dislocation structures were generally consistent with slip-system activity on the planes with slip traces [8].
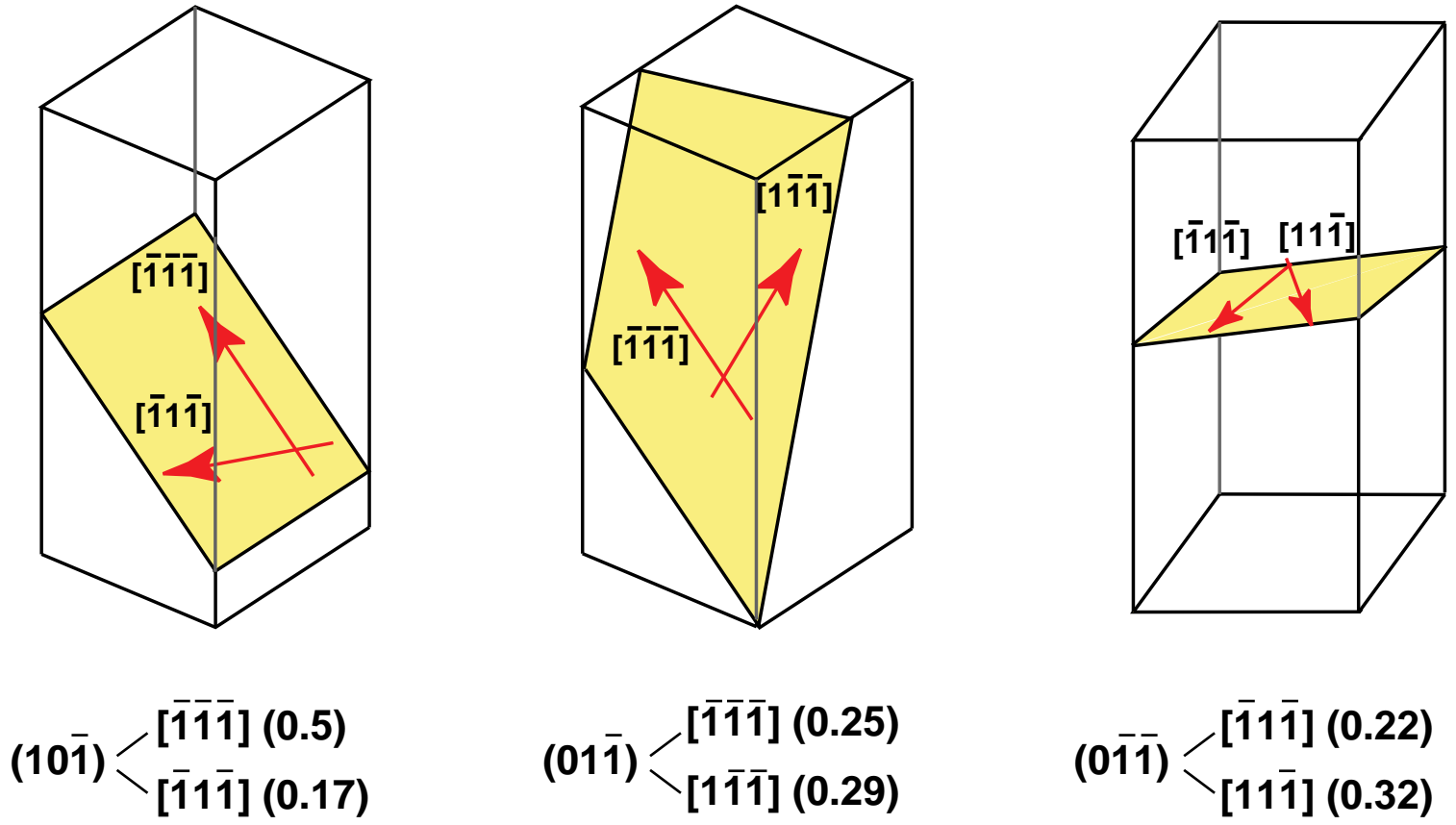

Figure 5. Optical light microscopy was used in conjunction with the Normarski interference

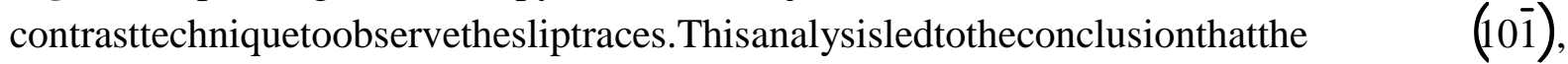
$(01 \overline{1})$, and $(0 \overline{1} \overline{1})$ slip planes, which are shown graphically, were active.

\section{CALCULATION OF SLIP SYSTEM ACTIVITY}

The goal of the analysis described in this section is to determine slip-system activity that is consistent with the experimentally determined strains (Figure 4) and the observed slip traces (Figure 5). The earliest description of this type of slip-system analysis was reported by Taylor [9], whose work was based on an energy criterion. Other approaches by Bishop and Hill [10] and Hutchinson [11] have also been published. The approach presented in this section follows the classical slip analysis that relates a general change in strain state to slip activity on five independent slip systems [12]. Our analysis focused on the six $\{110\}$ slip planes, each of which have two <111> slip directions for a total of 12 possible slip systems. There are 384 possible combinations of five independent slip systems of the $\{110\}<111>$ type that need to be considered. However, as our analysis shows, only one combination of slip systems is consistent with the experimentally observed slip traces.

The symmetric Schmid orientation tensor associated with a single slip system, $\alpha$, is given by

$$
\mathbf{M}^{\alpha}=\frac{1}{2}\left(b^{\alpha} \otimes n^{\alpha}+n^{\alpha} \otimes b^{\alpha}\right)
$$


where $b^{\alpha}$ is the slip direction defined by the Burgers vector and $n^{\alpha}$ is the slip plane normal related to the slip system $\alpha$. For the case of five slip systems, the deformation (or plastic strain) tensor becomes

$$
\begin{aligned}
& D_{i j}=\sum_{\alpha=1}^{5} \beta^{\alpha} \mathbf{M}^{\alpha} \\
& =\sum_{\alpha=1}^{5} \beta^{\alpha}\left|\begin{array}{ccc}
b_{1}^{\alpha} n_{1}^{\alpha} & \frac{1}{2}\left(b_{1}^{\alpha} n_{2}^{\alpha}+b_{2}^{\alpha} n_{1}^{\alpha}\right) & \frac{1}{2}\left(b_{1}^{\alpha} n_{3}^{\alpha}+b_{3}^{\alpha} n_{1}^{\alpha}\right)
\end{array}\right|
\end{aligned}
$$

where $\beta^{\alpha}$ is the amount of the crystallographic-glide strain, or slip-system activity.

Assuming zero volume change during plastic deformation at ambient pressure $\left(\varepsilon_{11}+\varepsilon_{22}+\varepsilon_{33}=0\right)$, we have a linear matrix system:

$$
\left.\left[\begin{array}{c}
\left.\varepsilon_{11}-\varepsilon_{33}\right\rceil \\
\varepsilon_{22}-\varepsilon_{33} \mid \\
\varepsilon_{23} \\
\varepsilon_{13} \\
\varepsilon_{12}
\end{array}\right]=\mathbf{B}\left|\begin{array}{l}
\left.\beta^{(1)}\right\rceil \\
\beta^{(2)}
\end{array}\right| \begin{array}{l}
\beta^{(3)} \\
\beta^{(4)} \\
\beta^{(5)}
\end{array}\right],
$$

where the terms in matrix $\mathbf{B}$ are geometrical factors dependent on the orientation of the slip systems. An inverse matrix exists for matrix $\mathbf{B}$ if the five chosen slip systems are independent. We can then write the slip-system activity as

$$
\beta=\mathbf{B}^{-1} \varepsilon
$$

In our analysis, all values are expressed with respect to the $x-y-z$ laboratory coordinate system, as shown in Figure 1. In addition, we chose the local "slip" coordinate systems such that the plastic work is always positive if the shear strain and shear stress have the same sign (i.e., both positive or both negative) (see Table I). The total plastic strain tensor was constructed from the strain gage data given in Figure 4. Average values for the lateral and shear strains are based on data from opposing sides of the sample cube, and the axial strain value is the average strain on all four sides. A plot of the total plastic strain as a function of time is shown in Figure 6. 
Table I. Slip-system index and Schmid Factors. The unshaded rows indicate slip systems that are consistent with observed slip traces.

\begin{tabular}{|c|c|c|}
\hline Index & Slip System & Schmid Factor \\
\hline 1 & $(01 \overline{1})[111]$ & 0.200 \\
\hline 2 & $(10 \overline{1})[111]$ & 0.500 \\
\hline 3 & $(1 \overline{1} 0)[111]$ & 0.250 \\
\hline 4 & $(01 \overline{1})[\overline{1} 11]$ & 0.287 \\
\hline 5 & $(\overline{10} \overline{1})[\overline{1} 11]$ & 0.470 \\
\hline 6 & $(\overline{1} \overline{1} 0)[\overline{1} 11]$ & 0.183 \\
\hline 7 & $(0 \overline{1})[1 \overline{1} 1]$ & 0.220 \\
\hline 8 & $(10 \overline{1})[\overline{1} \overline{1} 1]$ & 0.167 \\
\hline 9 & $(\overline{1} \overline{1} 0)[\overline{1} 1]$ & 0.053 \\
\hline 10 & $(0 \overline{1} \overline{1})[\overline{1} \overline{1} 1]$ & 0.317 \\
\hline 11 & $(\overline{10} \overline{1})[\overline{1} 1]$ & 0.197 \\
\hline 12 & $(1 \overline{1} 0)[\overline{1} \overline{1} 1]$ & 0.120 \\
\hline
\end{tabular}

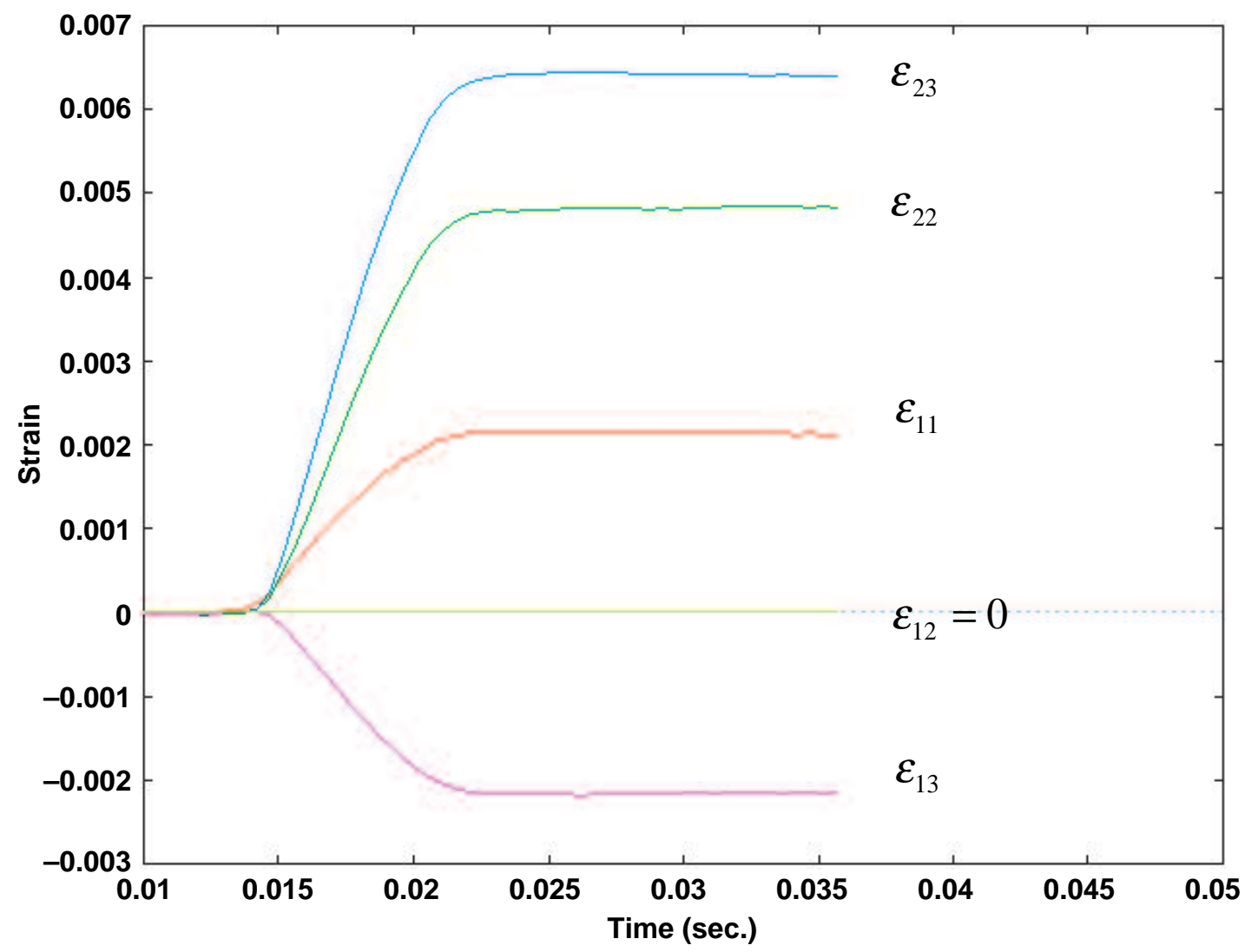

Figure 6. A time-history plot of strain components measured from experiments. The $\varepsilon_{12}$ strain was assumed to be zero; it is being measured in current ongoing experiments. 
The calculated slip-system activity must be consistent with the three slip planes that created

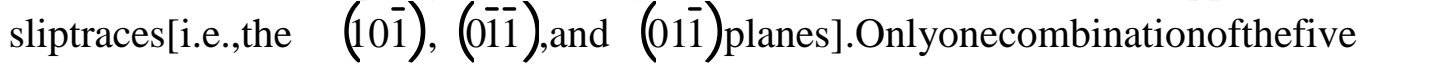
independent slip systems (out of all 384 possible groups) matched the observed slip traces: slip systems 2, 4, 7, 8, and 10 (see Table I for crystallographic indices). The resolved-shear-stress versus shear-strain curves determined by our analysis for these systems is shown in Figure 7. This solution satisfies the slip-trace analysis and the measured strain, but unfortunately, it does not include activity on slip system 1 , which is the [111] slip direction on the (011) plane. This is the so-called "anomalous" slip-plane and has a Schmid factor of 0.20. Despite this low Schmid factor, significant slip activity does occur on this plane. Ultimately, we need to determine how much slip activity occurs in both the $[111]$ and [111] directions. We are currently pursuing more elaborate analyses that will include a calculation of slip activity on all six slip systems. However, the results presented here provide a good starting point for the validation of DD simulations.

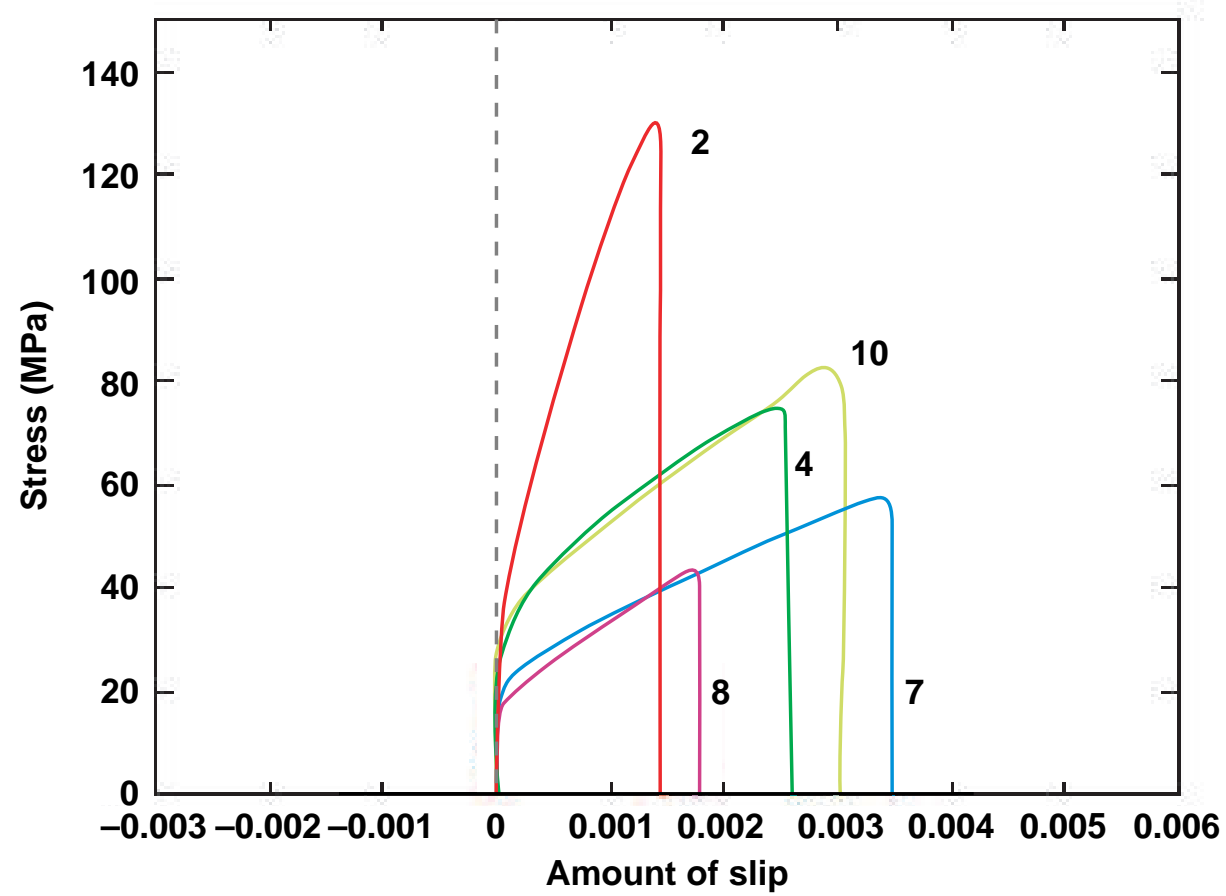

Figure 7. Plots of resolved-shear-stress versus shear-strain calculated from Eq. (5). The selected slip systems are 2, 4, 7, 8, and 10 (see Table I for index notation).

\section{VALIDATION OF DD SIMULATIONS}

At the current time, validation criteria for DD simulations have focused on uniaxial stressstrain response. This approach, which is 1-D in nature, has led to a critical study of both the temperature and strain rate dependence of bcc metals [13] and the effects of simulation boundary conditions on predicted work-hardening behavior [14]. The 6 DOF experiment described in this work was developed to provide experimental data for further validation of DD simulations. Current validation criteria based on the Mo experiments may include comparing the stress-strain 
response using 3-D strain information, the predicted slip-system yield, and work-hardening behavior. Key validation criteria are described below.

3-D Stress-Strain Response: The 6 DOF experiments allow essentially unconstrained deformation of a single crystal under a condition of applied uniaxial stress. A possible approach for the validation of DD simulations is based on using the experimentally determined 1-D stress history as input for the simulation. The predicted states of tensor strain for a given "stress history" could then be compared to experimentally determined strain states. This is essentially a 3-D validation of temporal stress-strain response.

Slip Planes: The slip-trace analysis of a test sample deformed to $0.6 \%$ axial strain has clearly identified three active slip planes. There could, of course, be slip activity on other planes. However, such slip activity would likely be minor in comparison to the slip activity that resulted in the observed traces. The results of DD simulations can be edited to determine slip-system activity. For the most part, the predicted slip activity should correspond to the experimentally observed slip planes.

Slip System Activity: The analysis in the Calculation of Slip System Activity section displays the extent to which slip activity and work-hardening behavior in five slip systems is consistent with the observed deformation. This information can be compared to DD simulation predictions. If this analysis is extended to include all six slip systems operating on the three observed slip planes, the comparison of this slip-system activity may be the most critical validation criteria. Our initial analysis of slip activity on the three active slip planes, as described in the previous section, is a good starting point for this comparison.

Dislocation Structures: Detailed TEM and X-ray topography of dislocation structures should also be compared to simulation results. For example, a thin region (on the order of $0.1 \mu \mathrm{m})$ can be "extracted" from a simulated dislocation structure and then allowed to relax using a free surface boundary condition. This DD result could then be compared to a TEM observation performed on thin foils harvested from deformed single crystals.

\section{DISCUSSION OF OBSERVED DEFORMATION RESPONSE OF MO SINGLE CRYSTAL}

The $[2, \overline{9}, \overline{20}]$ compression axis of the Mo single crystal test samples was selected to promote "single slip," with the primary slip system, (101) [111], having a Schmid factor of 0.5. However, the observed deformation response was found to be inconsistent with "single slip" on this slip system. If the sole cause of deformation had been slip activity in the primary slip system, the displacement of the lower platen with respect to the upper platen would have been in the negative $x$ direction, and there would not have been any displacement in the $y$ axis. The observed displacement in the $x$ direction was found to be opposite to this (i.e., positive $x$ displacement), and the displacement in the $y$ direction was substantially greater than the displacement in the $x$ direction. Future work is needed to understand this deformation response. It is interesting to note that the deformation of the test sample is such that the motion of the lower platen relative to the top acts to align the [001] pole with the compression axis of the test sample ( $z$ axis). Although the mechanism associated with this motion is unknown, this deformation promotes the most symmetric orientation with respect to slip activity in the bcc structure. 


\section{ACKNOWLEDGMENTS}

The authors would like to thank the following individuals for contributions to this work: Ms. R. Ann Bliss for X-ray characterizations, Mr. Robert Kershaw and Mr. Robert Vallier for metallography, and Mr. Barry Olsen for purification and heat treatment of the test materials. One of the authors (D. H. Lassila) would like to thank Dr. Howard K. Birnbaum and Dr. John P. Hirth for helpful discussions over the course of the work. We also wish to acknowledge discussions on the deformation response of bcc metals with Dr. Luke Hsiung, which motivated some of the experimental diagnostics.

This work was performed under the auspices of the U.S. Department of Energy by the University of California, Lawrence Livermore National Laboratory under Contract W-7405Eng-48.

\section{REFERENCES}

1. B. Devincre, V. Pontikis, Y. Brechet, G. Canova, M. Condat, and L. Kubin, "ThreeDimensional Simulations of Plastic Flow in Crystals," Microscopic Simulations of Complex Hydrodynamic Phenomena, edited by M. Mareshal and B. Holian (Plenum Press, New York, 1992), pp. 413-423.

2. H. Zbib, M. Rhee, and J. Hirth, "3-D Simulation of Curved Dislocations: Discretization and Long-Range Interactions," Advances in Engineering Plasticity and Its Applications, edited by T. Abe and T. Tsuta (New York, Elsevier Science, 1996), pp. 15-20.

3. K. Schwarz and J. Tersoff, "Interaction of Threading and Misfit Dislocations in a Strained Epiaxial Layer," Appl. Phys. Lett. 69, 1220-1222 (1996).

4. N. M. Ghoniem and M. Bacaloni, "Finite Segment Method for 3-D Dislocation Dynamics," University of California at Los Angeles, Los Angeles, CA, Eng. Report No. UCLA/MATMOD-97-01 (1997).

5. M. Rhee, D. Lassila, V. V. Bulatov, L. Hsiung, and T. Diaz de la Rubia, "Dislocation Multiplication in bcc Molybdenum: A Dislocation Dynamics Simulation," Phil. Mag. Letters 81(9), 595-605 (2001).

6. J. W. Christian, "Some Surprising Features of the Plastic Deformation of Body-Centered Cubic Metals and Alloys," Metall. Trans. (A) 14A, 1237-1255 (1983).

7. R. J. Arsenault, "Low Temperature of Deformation of bec Metals and Their Solid-Solution Alloys," Treatise on Mater. Sci. and Tech. 6, 1-99 (1975).

8. L. Hsiung, Lawrence Livermore National Laboratory, Livermore, CA, personal communication (2001).

9. G. I. Taylor, "Plastic Strain in Metals," J. Inst. Metals 62, 307-324 (1938).

10. J. F. Bishop and R. Hill, "A Theoretical Derivation of the Plastic Properties of a Polycrystalline Face-Centered Metal," Phil. Mag. 42, 1298-1307 (1951).

11. J. W. Hutchinson, "Plastic Deformation of bcc Polycrystals," J. Mech. Phys. Solids 12, 2533 (1963).

12. A. Kelly and G. W. Groves, Crystallography and Crystal Defects, (Harlow, U.K.: Longman Group, 1970), pp. 178-184.

13. M. Tang, L. P. Kubin, and G. R. Canova, "Dislocation Mobility and the Mechanical Response of bcc (Ta) Single Crystals: A Mesoscopic Approach," Acta Mater., 46, 32213229 (1998). 
14. V. Bulatov, W. Cai, and M. Rhee, Periodic Boundary Conditions for Dislocation Dynamics Simulations in Three Dimensions, edited by L. P. Kubin, J. L. Bassani, K. Cho, H. Gao, and R. L. B. Selinger (Mater. Res. Soc. Symp. 653, 2001). 